\title{
Axisymmetric collapses of granular columns
}

\author{
By GERT LUBE ${ }^{1}$, HERBERT E. HUPPERT ${ }^{2}$, \\ R. STEPHEN J. SPARKS ${ }^{1}$ AND MARKA. HALLWORTH \\ ${ }^{1}$ Centre of Environmental and Geophysical Flows, Department of Earth Sciences, \\ Bristol University, Bristol BS8 1RJ, UK \\ ${ }^{2}$ Institute of Theoretical Geophysics, Department of Applied Mathematics and Theoretical Physics, \\ Centre for Mathematical Sciences, University of Cambridge, Wilberforce Road, \\ Cambridge CB3 0WA, UK
}

(Received 30 July 2003 and in revised form 5 February 2004)

Experimental observations of the collapse of initially vertical columns of small grains are presented. The experiments were performed mainly with dry grains of salt or sand, with some additional experiments using couscous, sugar or rice. Some of the experimental flows were analysed using high-speed video. There are three different flow regimes, dependent on the value of the aspect ratio $a=h_{i} / r_{i}$, where $h_{i}$ and $r_{i}$ are the initial height and radius of the granular column respectively. The differing forms of flow behaviour are described for each regime. In all cases a central, conically sided region of angle approximately $59^{\circ}$, corresponding to an aspect ratio of 1.7 , remains undisturbed throughout the motion. The main experimental results for the final extent of the deposit and the time for emplacement are systematically collapsed in a quantitative way independent of any friction coefficients. Along with the kinematic data for the rate of spread of the front of the collapsing column, this is interpreted as indicating that frictional effects between individual grains in the bulk of the moving flow only play a role in the last instant of the flow, as it comes to an abrupt halt. For $a<1.7$, the measured final runout radius, $r_{\infty}$, is related to the initial radius by $r_{\infty}=r_{i}(1+1.24 a)$; while for $1.7<a$ the corresponding relationship is $r_{\infty}=r_{i}\left(1+1.6 a^{1 / 2}\right)$. The time, $t_{\infty}$, taken for the grains to reach $r_{\infty}$ is given by $t_{\infty}=3\left(h_{i} / g\right)^{1 / 2}=3\left(r_{i} / g\right)^{1 / 2} a^{1 / 2}$, where $g$ is the gravitational acceleration. The insights and conclusions gained from these experiments can be applied to a wide range of industrial and natural flows of concentrated particles. For example, the observation of the rapid deposition of the grains can help explain details of the emplacement of pyroclastic flows resulting from the explosive eruption of volcanoes.

\section{Introduction}

Granular media have been the focus of an increasing amount of research in recent years, because their properties and behaviour as either static piles or highly mobile flows are fundamental to the understanding of many man-made processes and natural phenomena. The deliberate flow of particulate matter is widely encountered in the industrial handling of grains and powders, while the mechanical stability of static piles of granular material is of central concern to many civil engineering projects. In nature, accumulations of non-cemented grains occur in both subaerial and subaqueous environments over scales ranging from millimetres to several tens of kilometres. Their tendency to remain statically packed or move is fundamental to phenomena ranging from the inexorable processes of erosion and sedimentation to the sudden catastrophic 
failures of landslides and generation of snow avalanches on mountains and pyroclastic flows in explosive volcanic eruptions.

In spite of these ubiquitous applications, even static problems involving granular media, such as the central issue of determining the pressure at the base of a stationary sand pile, are still controversial - see, for example, the reviews by Cates et al. (1999) or de Gennes (1999). Part of the difficulty stems from trying to describe quantitatively the forces set up between individual grains. In addition, the static friction against confining walls, if present, leads to a pressure with depth dependence that can be far from the hydrostatic relationship valid in a fluid.

Flowing granular media present further problems of great richness and complexity. While there are a variety of theories, and related governing equations, to describe particular aspects of granular motion, these (generally) continuum theories and equations are still deficient and much debated. Some progress has been made in considering the flow of granular material down chutes whose initial steepness is greater than the (static) angle of repose of the granular media, thus assuring its initial motion. The approach generally used by a variety of researchers has been to derive depthaveraged equations for flow down a rough inclined plane and introduce empirical friction factors to try to obtain agreement with data from experiments (see, for example Savage \& Hutter 1989; Anderson \& Jackson 1992; Savage 1998; Pouliquen 1999; Iverson \& Denlinger 2001, and some of the references therein). However, to quote Pouliquen (1999) "despite this apparent simplicity and the numerous experimental, numerical and theoretical works devoted to granular chute flows, their description and prediction are still a challenge". Some of the reasons why the challenge remains are described by Kadanoff (1999).

Many insightful reviews of the exciting and diverse areas of research into granular flows have appeared, including those by Campbell (1990), Savage (1995) and Jaeger, Nagel \& Behringer (1996).

The aim of the current work is to present experimental results for the fundamental problem of the collapse of initially vertical cylinders of granular material. The study focuses on the subsequent axisymmetric spreading of the dense granular flows. Aside from intellectual curiosity and the central nature of the problem, the study is primarily motivated by a need to understand the flow mechanics of granular material in the environment, such as occur in pyroclastic flows and debris avalanches. The study may also be relevant to understanding industrial granular flows generated either by accident or design. Pyroclastic flows and debris avalanches are some of the most destructive phenomena in nature. In order to assess their hazard potential, an understanding of their underlying physics is essential. Geophysical particulate flows are understood as multi-phase flows where volume, mass flux, grain size, particle concentration and bulk density can vary over several orders of magnitude (Druitt 1998). Thus, physical models describing the state of flow range from dense, granular flows, where the gas phase plays a subsidiary role (Dade \& Huppert 1998) and motion is dominated by particle interactions, through gas-fluidized flows, where gas plays a significant role (Roche et al. 2002), to highly diluted, turbulent systems where gas is the dominant phase and transports particles in turbulent suspension (Druitt 1998; Freundt \& Bursik 1998; Huppert 1998). Direct observations of such geophysical flows are rare and the study is often limited to making inferences from the characteristics of their resulting deposits. Interpretations of all such geological observations depend on a good understanding of the underlying fluid mechanics.

In contrast to classical fluid mechanics, general constitutive laws for granular flows are largely unknown and experimental studies in these circumstances provide a key 
approach to the investigation of their dynamical behaviour. Recent experimental studies on chute flows of granular matter (e.g. Savage \& Hutter 1989; Daerr \& Douady 1999; Pouliquen 1999; Pouliquen \& Forterre 2002) reveal interesting scaling laws on granular dynamics, but many of these experiments are performed under steady flow conditions. However, most of the dynamics in dense, geophysical flows are likely to involve unsteady motions. For example, many pyroclastic flows and debris avalanches are initiated on the steep flanks of a volcano and then run out and deposit on shallow slopes. The flows involve acceleration and deceleration and also stages of erosion and deposition.

In this paper we present the results of a series of experiments on axisymmetric granular flows in a simple set-up that (to our knowledge) has not been studied before. The experiments consist of suddenly releasing cylindrical columns of rough granular material over a flat surface, by rapidly raising the containing cylinder. The material then collapses radially and forms a symmetrical pile. The simplicity of this approach enables us to vary the initial parameters of the system (height and radius or volume of the column) systematically. Our aim in this study is twofold. First we are interested in the scaling behaviour for the flow motion and how the geometry of the resulting deposit varies with the initial parameters. To this end we have run experiments which change the initial geometry over at least 2 orders of magnitude. Once the simplest scenario of axisymmetric spreading of dense, well-sorted grains on a flat surface is understood, the set-up can be varied in the future to simulate situations closer to natural flows (e.g. by considering multiple grain sizes and densities, inclination of the ground, temperature etc.). Secondly, our experiments consider processes of flow deformation and sedimentation in dense granular flows. Experimental observations may help to understand the formation of sedimentary features in deposits of pyroclastic flows and debris avalanches that are still controversial. We note, by contrast, that if instead of rough grains we used smooth, frictionless spheres, the collapse would continue indefinitely. Alternatively, an ordered stack of regular shapes, such as a pile of bricks will not collapse at all.

The duration of each experiment was typically less than $2 \mathrm{~s}$ and detailed investigations of the flow process required the use of a high-speed video camera. This technique was employed in experiments conducted in Bristol University using sand as the granular material. A complementary set of experiments, aimed mainly at analysing the final geometries of axisymmetric collapses, was performed in Cambridge University, using predominantly sand and salt. Some additional experiments were conducted with couscous, rice, or sugar in order to investigate variations in grain shape and roughness.

The detailed descriptions of the experimental procedure, measurement methods and variations of experimental parameters are given in $\S 2$. Experimental observations are presented in $\S 3$. Results on the geometry of the final deposits of axisymmetric spreading flows are given in $\S 4$. In that section we also show how the geometry of the final deposits scales with the initial parameters and that the presented scaling laws change significantly within the large range of experimental parameters but little between the different grains. The dynamics of laboratory flows with sand are analysed using data on the motion of the flow front in $\S 4$. We present a scaling that describes the radial displacement of the flow front as a function of time for a range of initial parameters and compare this with the spreading of a fluid gravity current released instantaneously from a lock. Expressions for the total collapse time of the column are also obtained. A summary of our results and further discussion are presented in $\S 5$. Aside from our quantitative results describing the motion, the study points 


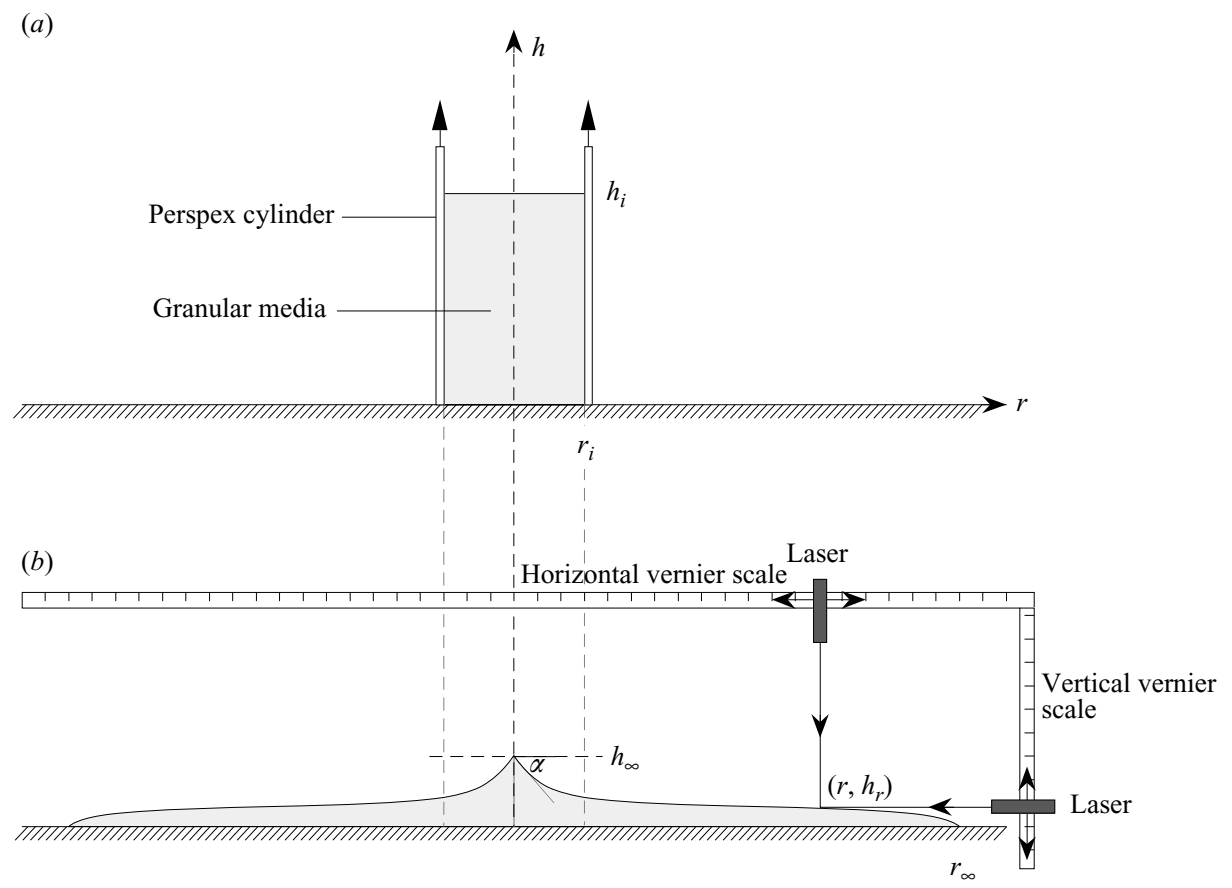

FIGURE 1. (a) Initial set up for the experiments and $(b)$ rough sketch of the geometry of the final deposit and how it was measured.

$\begin{array}{lccc}\text { Particle } & \begin{array}{c}\text { Mean density } \\ \left(\mathrm{g} \mathrm{cm}^{-3}\right)\end{array} & \begin{array}{c}\text { Mean grain size } \\ (\mathrm{mm})\end{array} & \begin{array}{c}\text { Angle of repose } \\ (\text { deg. })\end{array} \\ \text { Sand } & 2.6 & 0.32 & 30 \\ \text { Salt } & 2.16 & 0.30 & 30 \\ \text { Couscous } & 1.39 & 2 & 34 \\ \text { Rice } & 1.46 & 7 \times 2 & 31 \\ \text { Sugar } & 1.58 & 1 & 35\end{array}$

TABle 1. Properties of particles used in the experiments.

to the abrupt transition undergone between the flowing grains and the final static state, the only time during in which intergranular friction appears to be important in controlling the motion of the flowing material.

\section{The experiments}

\subsection{Experimental set-up}

The experiments were performed by rapidly releasing granular materials, initially contained in a partially filled cylinder, on to a flat surface and allowing them to spread out unhindered, as sketched in figure 1. Both dry sand with a narrow grain size distribution and vacuum-dried salt were primarily used as the particulate matter, and their density, mean grain size and angle of repose are given in table 1. Granular flows with salt were carried out on a smooth surface of baize. The sand flows were studied on three different surfaces: a smooth wooden plane, a smooth transparent 


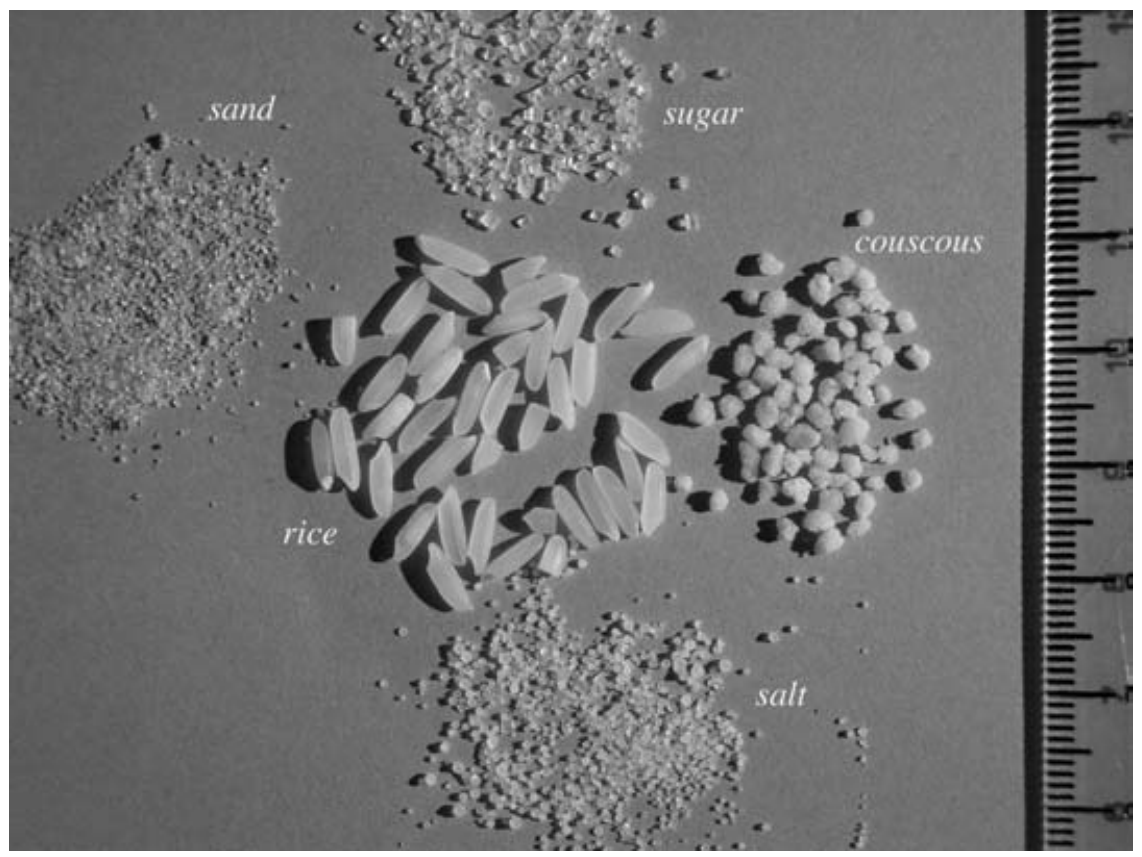

FIGURE 2. Photo of the five different grains used in the experiments.

Perspex plane and a rough plane made of sandpaper. The granular roughness of the sandpaper was of the order of the mean particle size of the granular flows. Additional experiments with couscous, rice and sugar were performed on both a smooth wooden plane and a smooth baize surface to further investigate interparticle friction for different grains. A photo of the different sets of grains, clearly showing their different geometrical shapes, makes up figure 2. All the results were independent of the different grains and the surface over which the grains flowed.

The initial aspect ratio $a$, given by the ratio of initial height, $h_{i}$, to radius $r_{i}$ was varied systematically by using different initial masses of material, $m_{i}$, and different diameter cylinders. The radii of the cylinders and the range of the initial aspect ratios used with each material are given in table 2. The initial columns were prepared by quickly pouring a measured mass of particles from above the centre of the cylinder. In order to produce a cylindrical shape the upper surface of the granular column was then flattened. A number of different ways of filling the cylinder were tested and they all lead to the same results. At the start of each experiment, the cylinder was quickly raised at velocities of around $2 \mathrm{~m} \mathrm{~s}^{-1}$ and the column collapsed and spread out to form a symmetrical pile, as sketched in figure $1(b)$.

\subsection{Measurement methods}

In order to investigate the dynamic behaviour of the granular motion, a digital high-speed video camera was used for all the experiments conducted in Bristol using sand. To preserve the images at reasonable sharpness, the experiments were recorded digitally at rates of 500 frames per second. Distance versus time data of the flow front were obtained from the footage by measuring the elapsed time at radial markings $1 \mathrm{~cm}$ apart. To study the flow and sedimentation processes at the base of the granular body, experiments were performed on a transparent Perspex plane and filmed from below. In order to help visualize the deformation and sedimentation processes during 


\begin{tabular}{|c|c|c|}
\hline Particle & Initial radius $(\mathrm{cm})$ & Range in $h_{i} / r_{i}$ \\
\hline \multirow[t]{3}{*}{ Sand } & 9.7 & $0.19-4.8$ \\
\hline & 7.45 & $0.44-4.62$ \\
\hline & 2.92 & $1.5-13.8$ \\
\hline \multirow[t]{5}{*}{ Salt } & 5.75 & $0.25-5.22$ \\
\hline & 3.19 & $1.09-17.7$ \\
\hline & 2.6 & $2.7-31.4$ \\
\hline & 2.25 & $2.0-35.0$ \\
\hline & 1.69 & $4.8-42.9$ \\
\hline \multirow[t]{3}{*}{ Couscous } & 7.3 & $0.31-1.89$ \\
\hline & 5.75 & $1.91-4.76$ \\
\hline & 2.55 & $9.10-25.1$ \\
\hline \multirow[t]{3}{*}{ Rice } & 7.3 & $0.44-1.99$ \\
\hline & 5.75 & $0.49-8.45$ \\
\hline & 2.55 & $5.22-25.3$ \\
\hline \multirow[t]{3}{*}{ Sugar } & 7.3 & $0.37-1.84$ \\
\hline & 5.75 & $1.86-3.69$ \\
\hline & 2.55 & $4.23-25.0$ \\
\hline
\end{tabular}

TABLE 2. Radius of cylinder and range of initial aspect ratio for each material.

flow, two experimental columns were prepared with zones of differently coloured particles. In the first experiment, three concentric zones were established, consisting of an inner cylinder of non-dyed particles, a middle ring of dyed particles and an outer ring of non-dyed particles. In the second experiment, the cylinder was filled with eight alternating horizontal layers of non-dyed and dyed particles. Both these experiments were carried out on a Perspex plane to study the base of the final deposit. For visualization of the internal deformation, some of the final deposits were cut by a thin transparent vertical Perspex plane.

After flow, measurements of the final runout $r_{\infty}$, the final cone height $h_{\infty}$, the steepest inclination of the pile $\alpha$ and a radial height profile through the pile were measured from the deposit using a point laser focusing technique, as shown in figure $1(b)$. In some cases, radial height profiles across the pile were also obtained using a penetrometer. This tool consists of thin wires $2 \mathrm{~cm}$ apart from each other and covered by a thin film of silicon grease. The wires are inserted vertically into the deposit down to its base, and then withdrawn. Heights were obtained by measuring the distance along which particles stuck to the wire. This method resulted in some destructive disturbance of the granular pile, and could not be used in areas where the angle of rest of the grains approached unstable values, particularly around the steep central cone. Heights obtained in this manner were accurate to $\pm 1 \mathrm{~mm}$, whereas heights and radii obtained by the laser point technique had errors estimated as $\pm 0.1 \mathrm{~mm}$. Repeatability of measurements for the final radius between experiments was of order $\pm 5 \mathrm{~mm}$.

\section{Flow description}

Our observations of axisymmetric granular flows from high-speed movies reveal the subdivision into three regimes of flow behaviour dependent on the initial aspect ratio. 


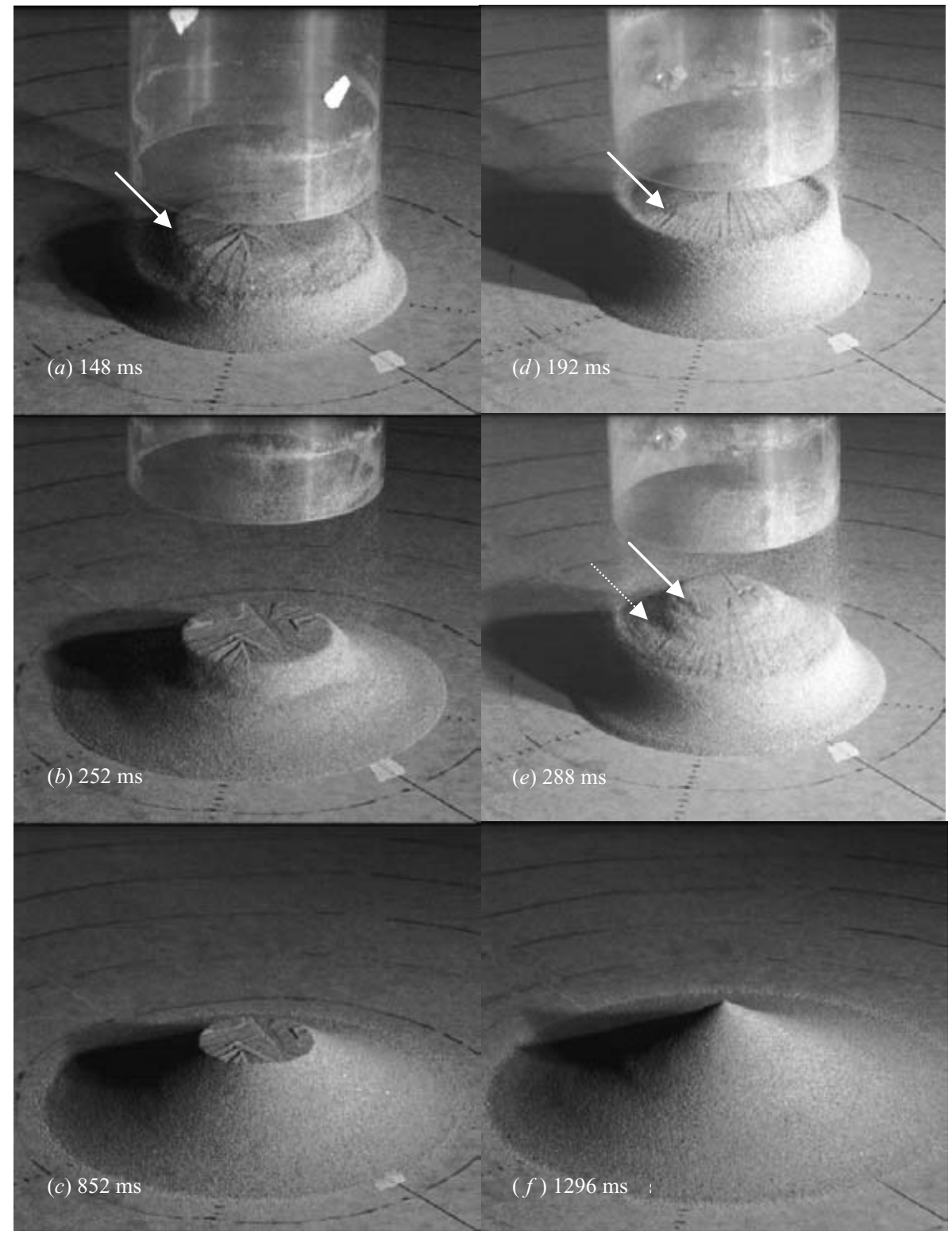

FiguRE 3. Two experiments, at three different times, with $a<1.7$. The photographs for the first experiment, with $a=0.55$, were taken at $(a) 148 \mathrm{~ms}$, (b) $252 \mathrm{~ms}$ and (c) $852 \mathrm{~ms}$, and are typical results for $a<0.74$. The photographs of the second experiment, with $a=0.9$, are at (d) $192 \mathrm{~ms},(e) 288 \mathrm{~ms}$ and $(f) 1296 \mathrm{~ms}$ and show the flow evolution for a typical experiment with $0.74<h i / r_{i}<1.7$. The arrows mark discontinuities in the slope of the free surface.

\subsection{Flows with aspect ratios less than 1.7}

For the situations with aspect ratios less than 1.7 (the reason for this limit is explained below), the free upper surface of the column divides into an inner, static axisymmetric region and an outer flowing region. Figure 3 depicts typical photographs for this situation. For flows with $a<0.74$ a single circular discontinuity on the surface of the column separates an outer, slumping region from a non-deformed inner region 
(figure $3 a-c$ ). For flows with $0.74<a<1.7$ the flow is more complex. Two circular discontinuities develop on the upper surface of the column, one rapidly after the other (figure $3 d$ ). Material beyond the first discontinuity starts flowing before material between the two discontinuities (figure $3 e$ ). The slope of the free surface gradually steepens from the flow front to the flat top, attaining the angle of repose there.

From photographs taken during the flow from below, three different regions were observed at the base: a static circular region, with the same radius $r_{i}$ as the initial container; a ring of previously deposited particles; and an outer ring of still flowing material. The last two regions are divided by a moving interface, which propagates outwards until the flow comes to rest. After the flow front stops, another moving interface, separating an outer static zone from an inner zone of flowing grains, was seen to propagate along the upper free surface from the stationary front towards the centre. The motion in the inner zone is in response to avalanching of the margins of the static circular region observed at the upper surface. This avalanching gradually reduces the radius of the inner zone. The extent of erosion of the circular region at the surface increases with increasing aspect ratio.

After all motion has ceased, for flows with $a<1$ a circular undisturbed area at the upper surface of the column, which remains at $h_{i}$, is preserved (figure $3 c$ ), connected to a slope with maximum inclination approximately at the static angle of repose $\left(\alpha=32^{\circ}-32.5^{\circ}\right.$ for sand). In contrast, late-stage avalanching for $1<a<1.7$ erodes the entire initial column surface leaving a conical pile at the peak (figure $3 f$ ). The maximum inclinations for flows with $1<a<1.7$ were between $3^{\circ}$ and $5^{\circ}$ less than the static angle of repose.

\subsection{Flows with intermediate initial aspect ratios}

For columns of $a>1.7$ (figure $4 a$ ), the entire upper surface starts to flow immediately. At the base of the column a flow front develops and axisymmetrically propagates outwards (figure $4 b$ ). In the first stage of flow the upper free surface of the column remains undeformed and horizontal (figure $4 b$ ). After the column has lost some height, deformation of the top occurs to form a dome whose radius of curvature decreases with time (figure $4 c, d$ ). Just before the central body relaxes towards its final cone-like shape, two concentric stationary bulges appear on the surface around its steep slope (white arrows in figure $4 e$ ). Additionally, as the flow front decelerates, two outwardly propagating concentric surface waves develop across the free surface (black arrows in figure $4 e$ ). Their amplitude of a few millimetres is relatively small compared to their wavelength of $2-3 \mathrm{~cm}$. From the time the flow front stops, a moving interface between deposited and flowing grains propagates inwards across the free surface from the flow front to the central cone (figure $4 f$ ). This surface phenomenon eliminates the waves and they are not preserved in the final deposit. As with flows for $a<1.7$, there are three regions at the base of the flowing granular body: an originally static circular region with radius $r_{i}$; a ring of previously deposited particles; and an outer ring of still moving particles. In this outer ring, particle velocities increase with radial distance. For most of the experiment the basal interface between deposited and moving particles continuously propagates outwards. As the flow front decelerates, the interface between deposited and moving grains abruptly jumps to a position further out. After the jump, the new interface between deposited and moving particles becomes wavy. A second jump of the interface between deposited and moving particles occurs just before the flow front stops. The radial distances of the regions before and after the jumps were measured in the high-speed movies. They coincide with the radial distances of the troughs of the concentric waves at the free surface. 


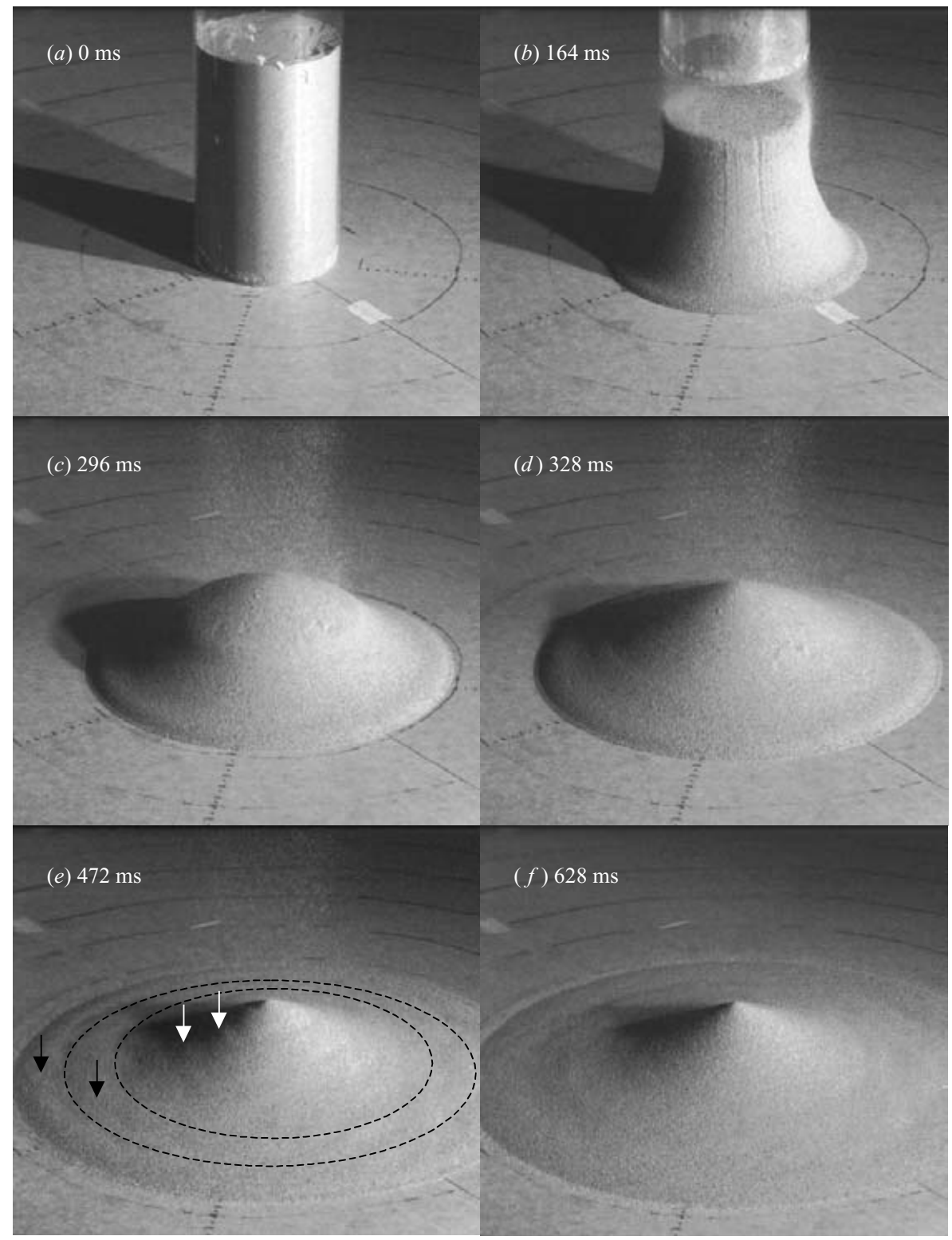

FIGURE 4. Flow evolution at various times for an experiment for which $a=2.75$.

The final deposit had a steep central cone and an axisymmetric tapering frontal region. The maximum inclination $\alpha$ of the deposit was slightly smaller than the static angle of repose. Flows with $1.7<a \leqslant 4.6$ show similar inclinations $\left(\alpha=25.5^{\circ}-27^{\circ}\right.$ for sand).

When the experiment with three marked concentric zones was vertically sectioned (figure 5) an originally static, central cone-like body can be observed. The most distal parts of the deposit originate from the outermost initial concentric zone. The middle zone has slumped closer to the original position, but is always separated from the ground by a thin zone (a few grain diameters) with particles from the outer zone. 

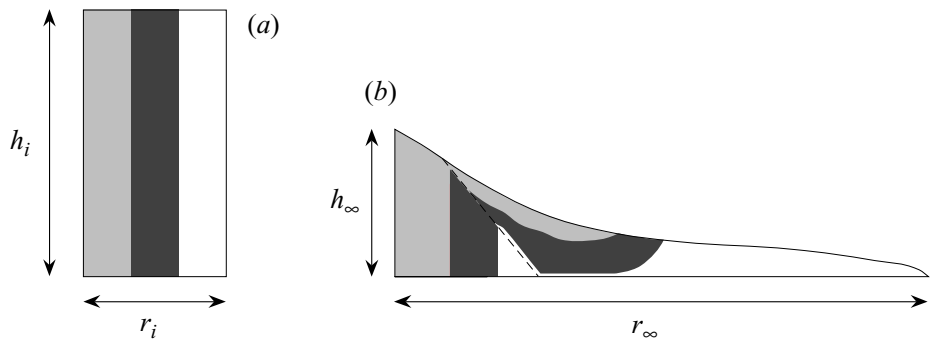

FIGURE 5. A vertical section of the deposit $(b)$ of a column with three initially concentric zones $(a)$.

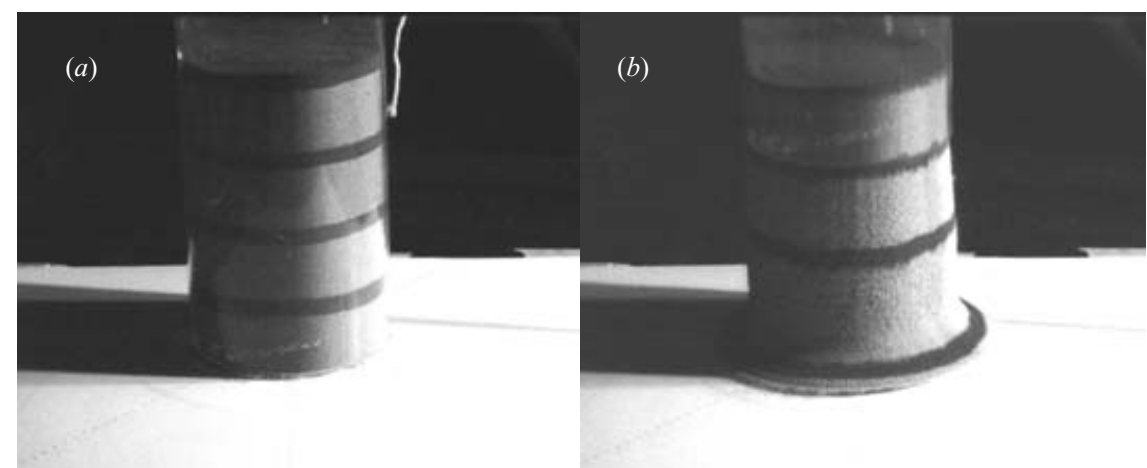

(c)

$(d)$

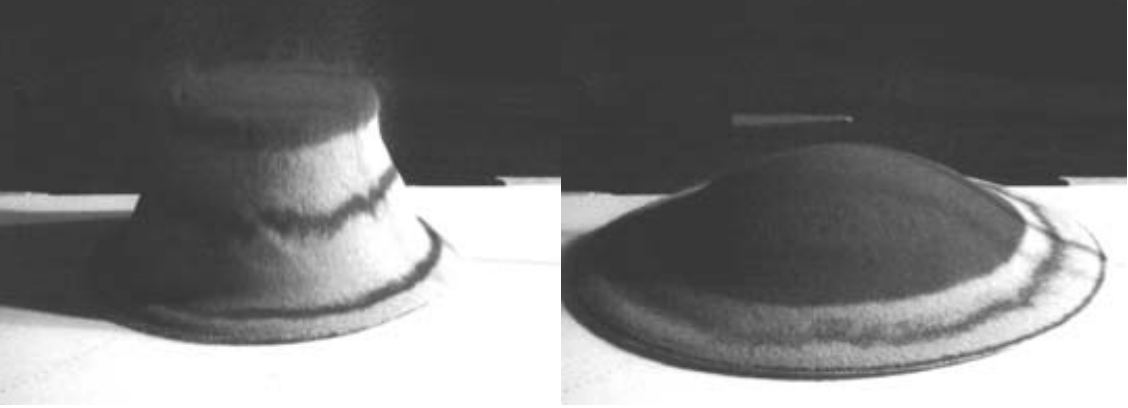

FIGURE 6. Sequence of photographs from an experiment with four marked horizontal layers and $a=3.0$.

This implies that the interface between deposited and moving particles evolves into the flowing body as a shallow plane.

Qualitative observations of grain motion along the free upper surface can be deduced from experiments with a column of initially marked horizontal layers. From the top of the column towards the flow front, the free surfaces of these layers are first increasingly stretched in the flow direction at the steep inclined part of the failing column and then increasingly compressed at the outer, flat tapering region (figure 6). The stretching and compression of the free surfaces of these layers is due to different particle velocities at different positions along the free surface. Thus, as observed for the flow front motion, the velocity of particles along the upper free surface change with time. Additional experiments with specially placed marker grains indicated that grains initially in the uppermost region ended up blanketing most of the final pile. 
Also, of those grains initially on the vertical margins of the cylinder, all but those in a small region near the top ended up at the base of the deposit. This parallels the fluid mechanical concept that in free flow a fluid parcel which starts on a surface remains on a surface. The experiments with marker beds also indicated that these granular flows do not involve much mixing between initially horizontal, different layers, which become deformed and distorted but not mixed during their displacement.

\subsection{Flow with large initial aspect ratios}

The flow behaviour of the collapse changes as the initial aspect ratio increases; and our data suggest that another transition occurs at around an aspect ratio of roughly ten. After lifting the container, the entire free surface starts to flow immediately. A flow front develops at the basal margin of the column and propagates radially as a thin sheet (figure $7 b$ ). In contrast to low-aspect-ratio flows, the upper surface of the column remains undeformed until its height is reduced to that of the neighbouring flat frontal region (figure $7 c, d$ ). From that time a concentric wave originates from the margin of the falling column and propagates outwards (figure $7 d$ and $e$ ). Since the wave moves at greater velocity than the flow front it eventually reaches the front, whereupon a thickening of the flow front is observed. Before the flow front has stopped, a moving interface between an inner zone of static grains and surrounding flowing grains propagates along the free surface from the cone towards the front (figure $7 f$ ).

As with flows of lower initial aspect ratios, three different regions were observed at the base of the flow: an originally static circular area with radius $r_{i}$; a ring of previously deposited particles; and an outer ring of moving particles. In the first phase of the experiment the interface between deposited and flowing grains continuously propagates outwards. Approximately from the time the wave develops at the free surface, this interface propagates backwards. Thus, already deposited particles are remobilized. A few tens of milliseconds later the direction changes again and the interface between deposited and moving particles propagates outwards. As with flows of intermediate initial aspect ratios, the interface can then jump abruptly outwards. After the jump the interface continuously propagates outwards until a second jump appears a few centimetres behind the flow front.

The final deposit shows a small central cone and a tapering frontal region. The maximum inclination $\alpha$ at the cone is much smaller than the static angle of repose. Our data suggest that $\alpha$ decreases with increasing initial aspect ratio. Much of the remainder of the deposit is of almost uniform thickness. Two concentric bulges were preserved, one near the flow front and the other approximately at half of the runout distance. The bulges were centred above the radial positions where the jumps of the interface between deposited and moving grains were observed at the flow base near the end of emplacement.

\section{Results}

\subsection{Geometry of the inner, static body}

When viewed from below, a static region with radius $r_{i}$ was observed in all sand experiments carried out in Bristol. For flows with $a<1.7$ a circular discontinuity between a static and a surrounding flowing region was observed at the upper surface of the column. The size of this static region was measured in the high-speed movies after its first appearance at the radial distance $r_{a}$. There is a good linear relationship between $r_{a}$ and $h_{i}$, the height of the static region at the upper surface of the column, 


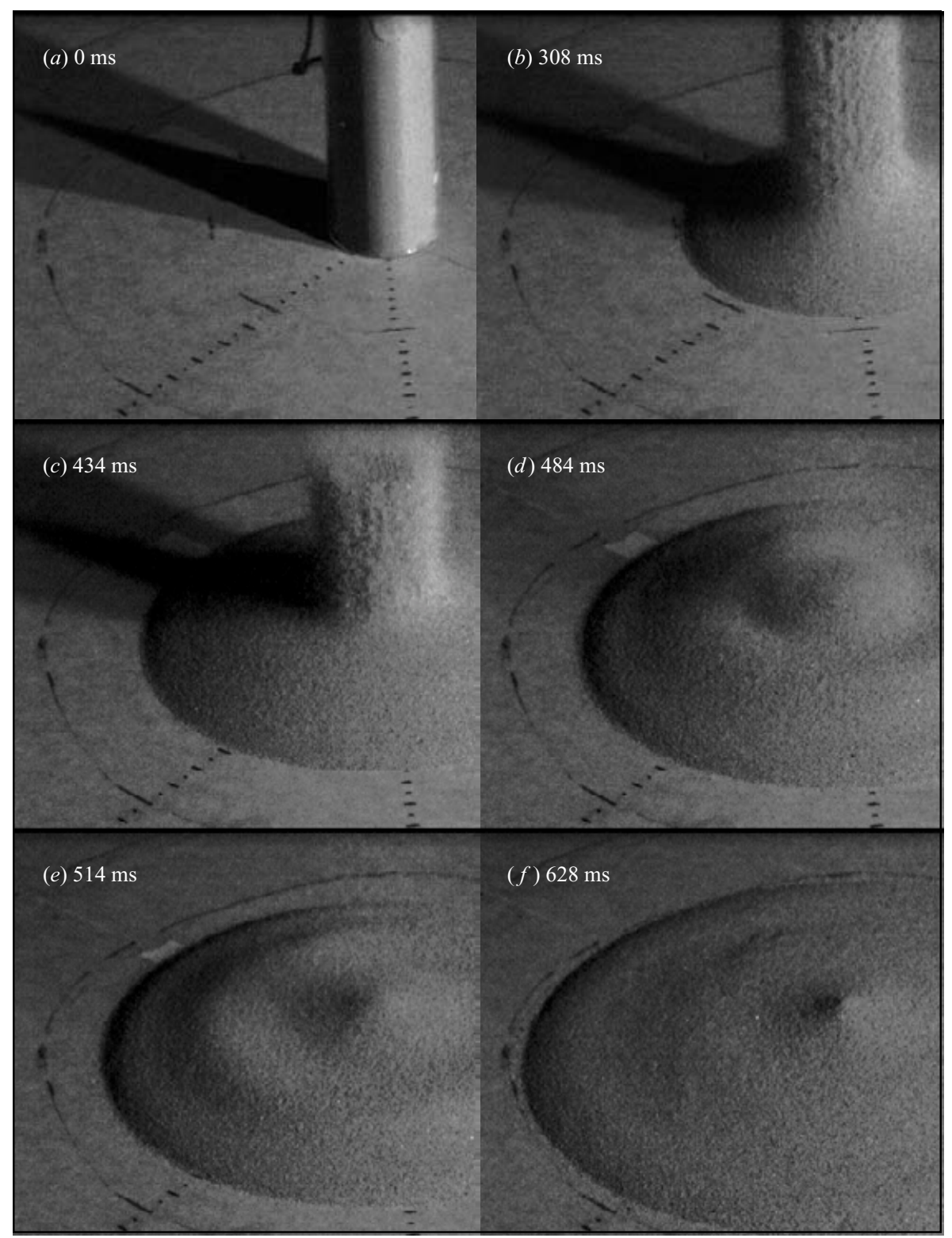

FIGURE 7. Flow evolution at various times from an experiment with $a=13.8$.

for cylinders with radii $r_{i}=9.7 \mathrm{~cm}$ (figure 8 ). The regression line through the data indicates a zero height at a radial distance of $9.64 \mathrm{~cm}$, satisfyingly close to $r_{i}=9.7 \mathrm{~cm}$. This result is consistent with an inner, static body with a cone geometry. The outer inclination of that cone, calculated from the regression line, corresponds to an angle of $59.4^{\circ}$, which can be interpreted as an internal friction angle for the sand. The aspect ratio of this cone is $\tan ^{-1} 59.4^{\circ}=1.7$.

For flows with $a>1.7$ no discontinuity was observed at the upper surface of the column. However, the relaxation of the central region towards a cone-like shape suggests a static interior in the centre. Cutting a deposit of a column with marked layers shows the geometry of this inner, static body to be a steep inclined cone with 


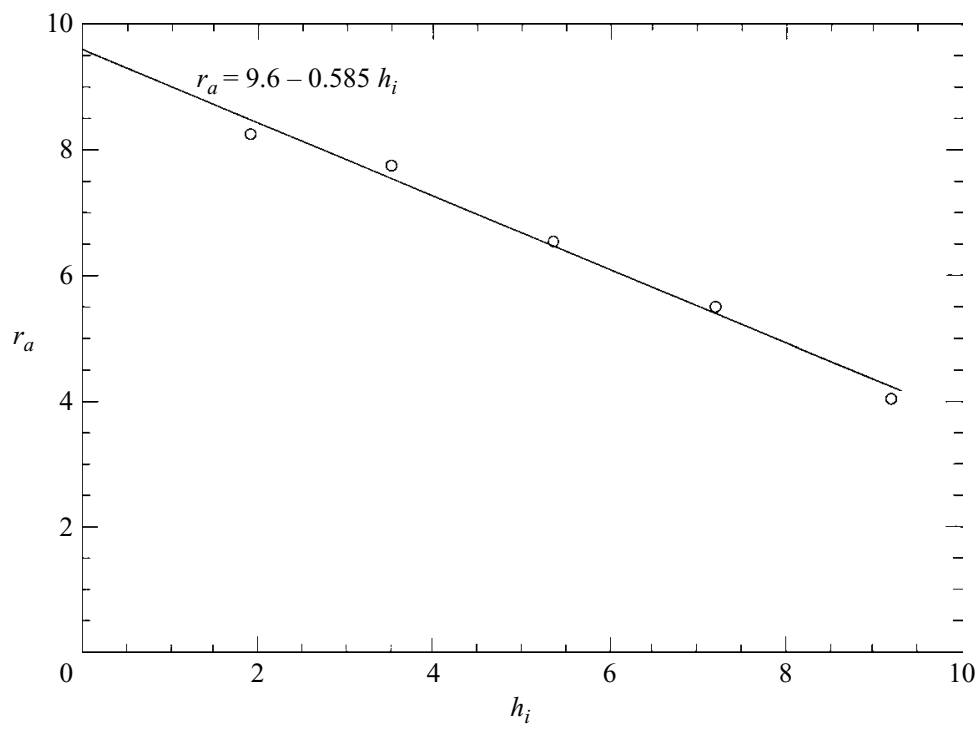

FiguRE 8. The radius of the undisturbed free surface, $r_{a}$, as a function of the initial height, $h_{i}$, from experiments on sand with $a<1.7$. The best-fit straight line through the data is also presented.

a slightly flattened top (figure 5), which is consistent with the partial erosion of the primary inner, static body by the flow.

\subsection{Scaling laws for the final runout}

The initial experimental set-up is uniquely expressible in terms of any two of the variables $r_{i}, h_{i}$ and $V=\pi r_{i}^{2} h_{i}$. Thus, under the assumption that no internal parameters, such as the value of the Coulomb friction between grains, are relevant during the flow, the final runout radius, $r_{\infty}$, and height, $h_{\infty}$, can also be written in terms of any two of these three variables. Put another way, the success with which we can quantitatively describe all our experimental data, for different grains, in terms of two of these variables only supports the validity of the assumption that no other parameter plays an essential role. Further confirmation of this result comes from the kinematic data discussed in $\S 4.6$.

Our observations that the form of the collapses differs with different aspect ratios $a=h_{i} / r_{i}$ suggests using $a$ as a fundamental parameter, to which we add, for convenience, $r_{i}$. (Either $h_{i}$ or $V_{i}$ could also be used to lead to the same results.) Then, by dimensional analysis, we can write

$$
r_{\infty}=r_{i} \tilde{f}(a)
$$

where $\tilde{f}(a)$ is a (non-dimensional) function of the aspect ratio, to be determined from our experiments (or theory). It turns out in what follows that it is more convenient to express (1) in the slightly different, but equivalent, form

$$
\frac{r_{\infty}-r_{i}}{r_{i}}=f(a)
$$

where it is now the unknown (non-dimensional) function $f(a)$ which is sought (and related to $\tilde{f}(a)$ by $f(a)=\tilde{f}(a)-1)$. Part of the reason to prefer the use of $f(a)$ is 


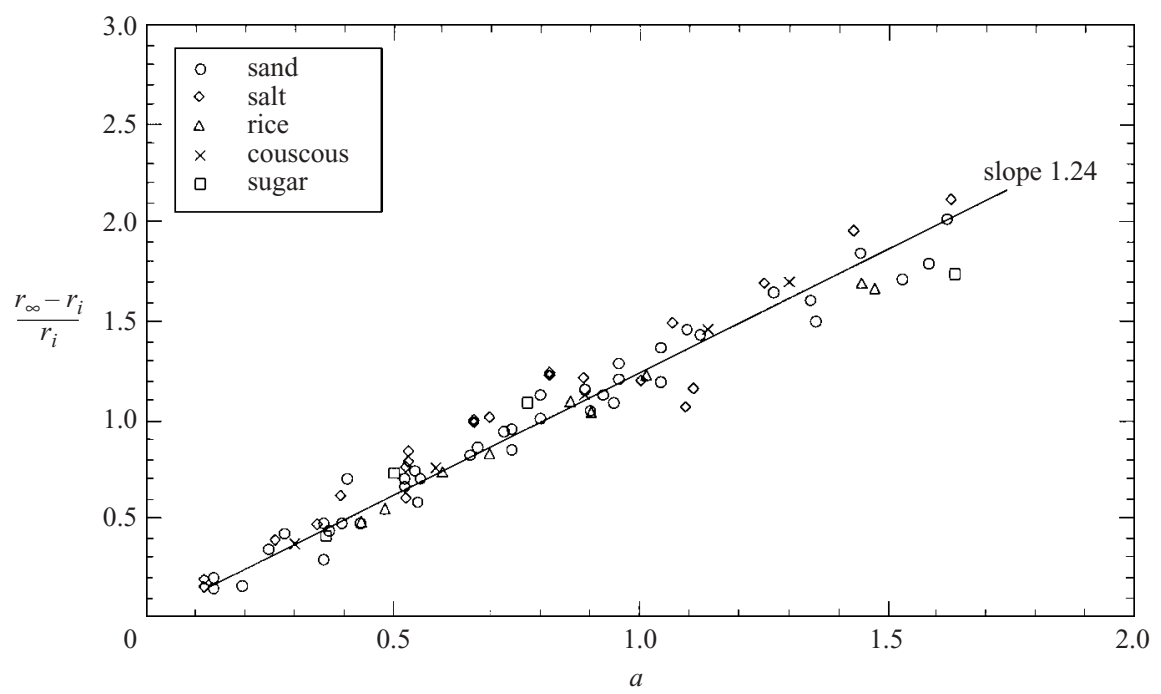

FIGURE 9. The non-dimensional radial excursion, $\left(r_{\infty}-r_{i}\right) / r_{i}$, as a function of the aspect ratio $a$ with $a<1.7$ for sand, salt, rice, couscous and sugar. A straight line through the origin at slope 1.24 has a regression coefficient of 0.975 .

that for a very low column $r_{\infty}-r_{i} \rightarrow 0$ and so $f(a)=0$, i.e. the function sought goes through the origin.

In the flow regime with $a<1.7$, the functional form of $f(a)$ can be determined by either physical or mathematical reasoning. Both arguments depend on the fact that, in this range, since only grains initially beyond a specific radius, $r_{a}$, take part in the motion, the value of $r_{i}$ itself must be irrelevant except in relation to the displacement of the cylindrical edge, $\delta r \equiv r_{\infty}-r_{i}$. This displacement is a length and the only length to which it can be related is $h_{i}$, and by dimensional analysis the relationship must be linear. Thus $\delta r=c h_{i}$, for some constant $c$. Or in terms of (2)

$$
f(a)=c a \quad(0 \leqslant a<1.7) .
$$

The complementary, mathematical argument is that the only way (2), expressed as $\delta r / r_{i}=f\left(a=h_{i} / r_{i}\right)$, can be independent of $r_{i}$ is if $f(a)=c a$.

In figure 9 we plot all our data for all the different grains for $\delta r / r_{i}$ as a function of $a$. We see that they are represented well by a straight line, with $c=1.24$ (for all materials).

We plot all our data for $\delta r / r_{i}$ in figure 10 on logarithmic axes. For the range of collapses with $1.7<a$, a good power-law representation of the data is given by

$$
f(a)=1.6 a^{1 / 2}
$$

(for all materials).

Equation (4) is the major quantitative result of our investigation, and can be written as

$$
r_{\infty}=r_{i}+0.90\left(V / r_{i}\right)^{1 / 2}
$$

A theoretical determination of (5), which indicates that the radial spread increase with the square root of the volume, remains a challenge for the future.

Note that the data plotted in figure 10 include those taken from flows on sandpaper. The similarity of the results with rough and smooth surfaces confirms that the interaction of the flow with the ground suface makes little difference to the runout. 


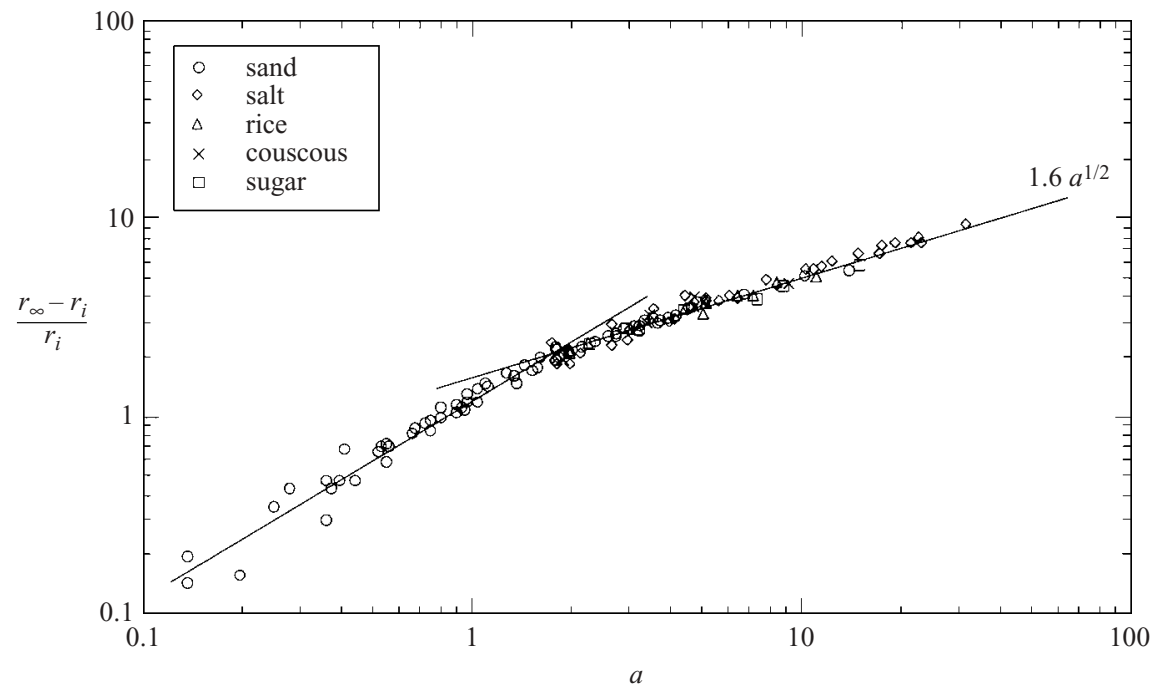

FIGURE 10. The non-dimensional radial excursion, $\left(r_{\infty}-r_{i}\right) / r_{i}$ as a function of the aspect ratio $a$ (on logarithmic axes). The curve $1.6 a^{1 / 2}$ is the best-fit half-power law through the data for $a>1.7$ with a regression coefficient of 0.988 .

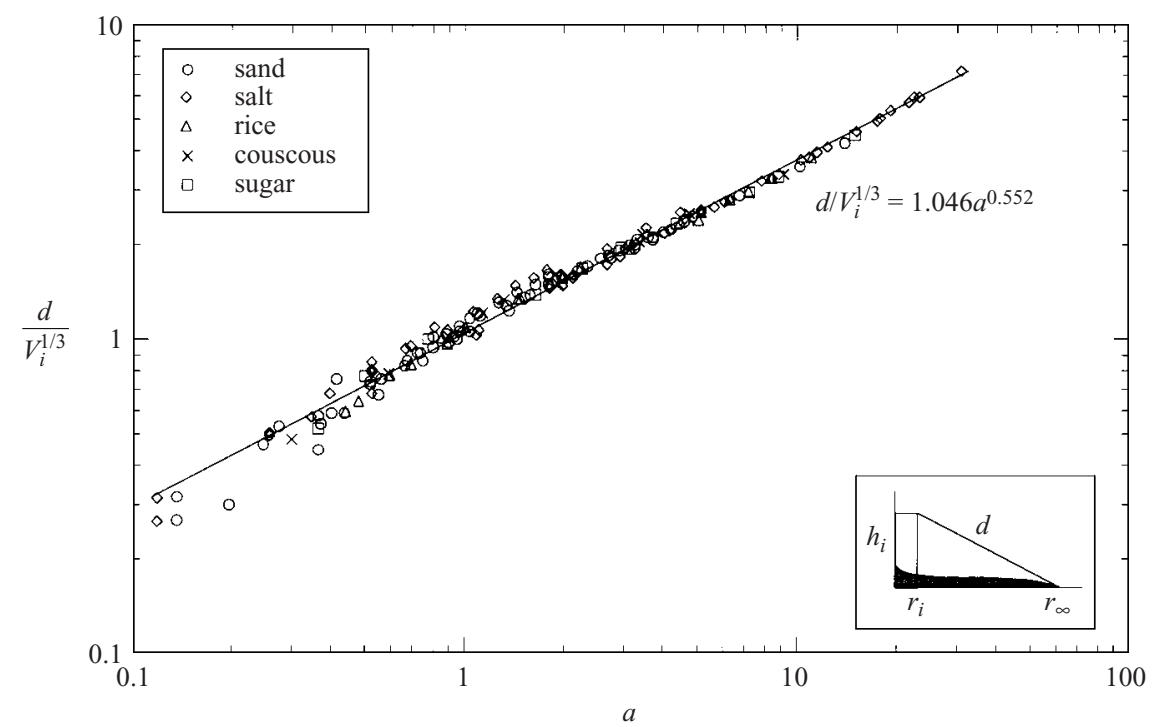

FIGURE 11. Data for $d / V_{i}^{1 / 3}=\left[\left(r_{\infty}-r_{i}\right)^{2}+h_{i}^{2}\right]^{1 / 2} / V_{i}^{1 / 3}$ as a function of the aspect ratio $a$ with the best curve fit $d / V_{i}^{1 / 3}=1.05 a^{0.55}$ and an inset to define $d$.

This is consistent with the interpretation, based on the formation of the broad layer in figure 6, that a dynamic interface develops within a few particles of the base and separates the flow between deposited and flowing material.

While analysing the salt and sand data for the final runout length, we noticed that the length of the straight line from a point on the top perimeter of the initial cylinder of granular material to the furthest point on the base (see inset of figure 11)

$$
d=\left[\left(r_{\infty}-r_{i}\right)^{2}+h_{i}^{2}\right]^{1 / 2}
$$




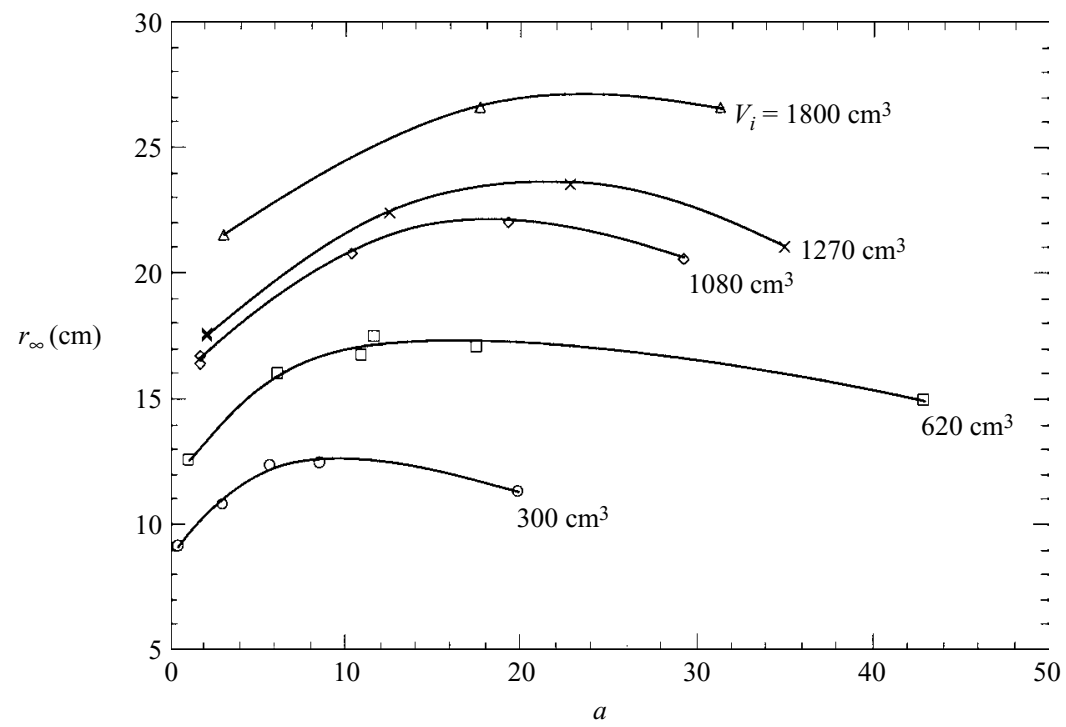

FIGURE 12. The (dimensional) final runout, $r_{\infty}$, as a function of the initial aspect ratio, $a$, for various experiments with constant volumes. There is a tendency for $r_{\infty}$ to slightly decrease for large a.

was accurately represented by $1.05 V_{i}^{1 / 3} a^{0.55}$, which is expressible (with a little algebra) as

$$
r_{\infty}=r_{i}\left[1+\left(2.36 a^{1.77}-a^{2}\right)^{1 / 2}\right] .
$$

The result is displayed in figure 11. Even though (6) gives a simple continuous representation of the data, it cannot be appropriate for large $a$, because while the right-hand side of (6) is a monotonically increasing function of $a$ until $a=27$, thereafter it begins to decrease until at $a=46$ it is zero. However, we notice that plotting $r_{\infty}$ as a function of $a$ for constant volumes (figure 12) tentatively suggests an increase for smaller values of $a$ and a tendency to decrease beyond that, at a value of $a$ which increases with $V_{i}$, but our data are sparse in this region.

\subsection{Final cone height}

The final cone height is dependent on the initial height and aspect ratio and is a consquence of both the collapsing and subsequent avalanching. The relationship for the final cone height on the basis of dimensional analysis is of the form

$$
h_{\infty}=r_{i} \phi(a)
$$

where $\phi(a)$ is a non-dimensional function of $a$.

For $0 \leqslant a<1.7, h_{\infty}=h_{i}$, which indicates that

$$
\phi(a)=a \quad(0 \leqslant a<1),
$$

which is consistent with the argument that in this range of $a, h_{\infty}$ is independent of $r_{i}$.

For $1.7 \leqslant a<10$, all our data, plotted on figure 13 , are represented well by

$$
\phi(a)=0.88 a^{1 / 6} \quad(1.7<a<10)
$$




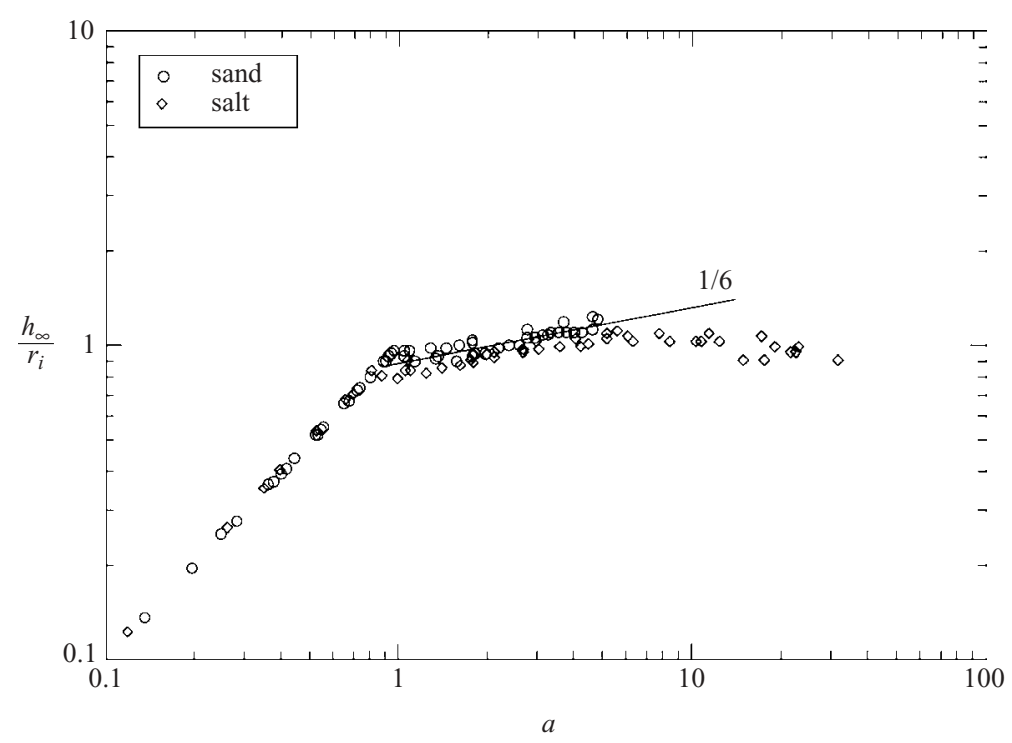

FIGURE 13. The non-dimensional final height at the centre of the granular pile, $h_{\infty}$, as a function of the aspect ratio $a$. The data between $a=1.7$ and $a=10$ have a slope of $1 / 6$.

or alternatively,

$$
h_{\infty}=0.73\left(V r_{i}^{3}\right)^{1 / 6} \quad(1.7<a \lesssim 10) .
$$

These coefficients are mean values, with some slight systematic deviations of approximately $10 \%$ for salt and sand. This variation is thought to reflect the different influences of intergranular friction on late-stage avalanching.

For $a>10$ there is an indication that $h_{\infty}$ decreases with increasing $a$, as shown on the graph. This is probably an effect of the wave that originates from the centre and with increasing $a$ increasingly transports material from the centre.

\subsection{Height profiles}

The shape or height profiles of the deposit are dependent on the value of $a$. Using dimensional arguments, the contour of the final deposit must be expressible as

$$
h(r) / h_{\infty}=\eta\left(r / r_{\infty}, a\right)
$$

where $\eta$ is a dimensionless function of its two dimensionless variables with, by definition,

$$
\eta(0, a)=1 \quad \text { and } \quad \eta(1, a)=0 .
$$

Figure 14 plots $h(r) / h_{\infty}$ as a function of $r / r_{\infty}$ for several values of $a$. A test of any theoretical model to describe column collapse would be its agreement with the data of this figure.

Determining the appropriate integrals using the data displayed in figure 14, we confirm that the final volume of the granular pile equals its initial volume, so that there is no net volume inflation. Determining the final surface area of the free surface $S_{\infty}$, non-dimensionalized with respect to $\pi r_{i}^{2}$, we obtain a graph which 


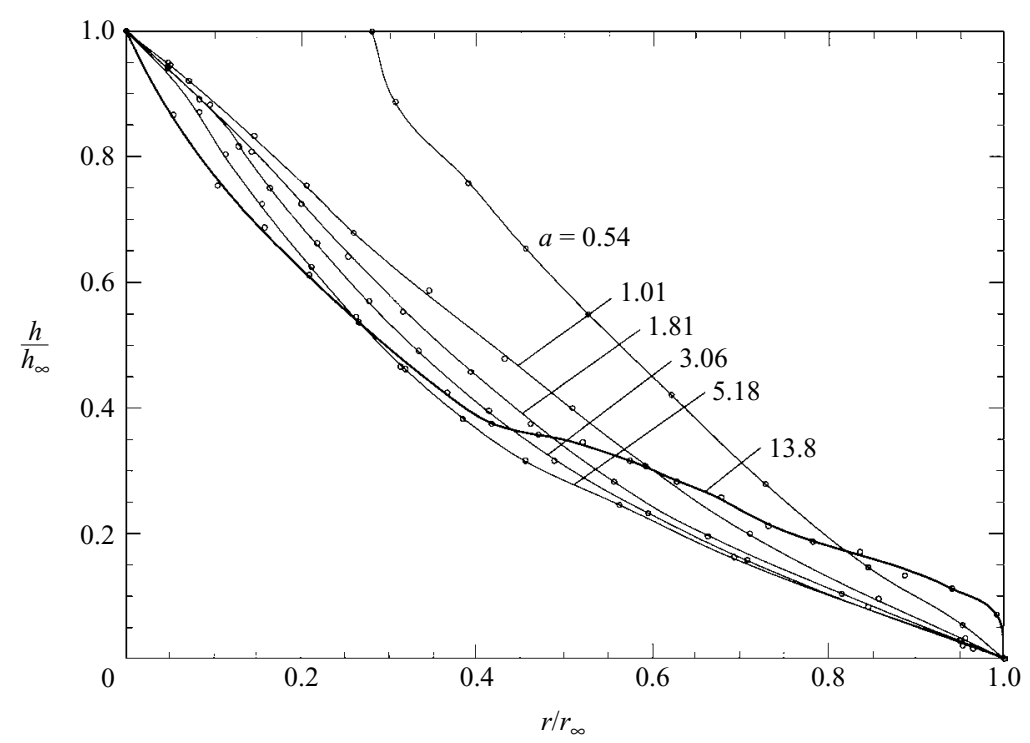

FIGURE 14. The non-dimensional height of the final profile as a function of non-dimensional radius for different aspect ratios.

approximates 1 for small $a\left(r_{\infty} \approx r_{i}\right.$ and so $\left.S_{\infty} \approx \pi r_{i}^{2}\right)$ and increases linearly with $a$ for large $a\left(S_{\infty} \approx \pi r_{\infty}^{2}\right)$.

\subsection{Further experiments}

We have also conducted some preliminary 'drop' experiments for which the cylinder of granular material was raised by a height $H$ off the base and then dropped, to fall to the base and then spread axisymmetrically. All the material dropped a height $H$ under gravity, whereafter the bottom of the pile hit the base and radial spreading commenced. Preliminary results of the non-dimensionalized radial displacement $\left(r_{\infty}-r_{i}\right) / r_{i}$ and the final central height $h_{\infty} / h_{0}$ are plotted as functions of an extended aspect ratio $a_{H} \equiv\left(H+h_{i}\right) / r_{i}$ in figure 15 which includes all the previous data with $H=0$. The majority of the new values of $a_{H}$ are large, in the third aspect-ratio regime. Rather surprisingly, the results for $r_{\infty}$ and $h_{\infty}$ dovetail well with the results for $H=0$, although the shape of the deposits must be quite different. This suggests that allowing the granular material to drop before it hits the ground introduces no new phenomena (other than the initial downward acceleration under gravity) and allows an effective aspect ratio $a_{H}$, which is much larger than the aspect ratio of the initial cylinder, to be investigated. The result is also consistent with the idea that the final extent of the deposit is reached by grains in the uppermost region of the initial cylindrical pile. We plan to present further results of this case in a subsequent publication.

\subsection{Kinematic data}

In order to understand the dynamic behaviour better we analysed the data collected of the radius $r$ of the flow front as a funtion of time $t$. Three different patterns were observed, dependent on the initial aspect ratio (figure $16 a-c$ ). For flows with aspect ratios less than 1.7 there was a primary acceleration phase followed by a deceleration phase (figure 16a). Flows with $1.7<a<10$ had an acceleration phase, 


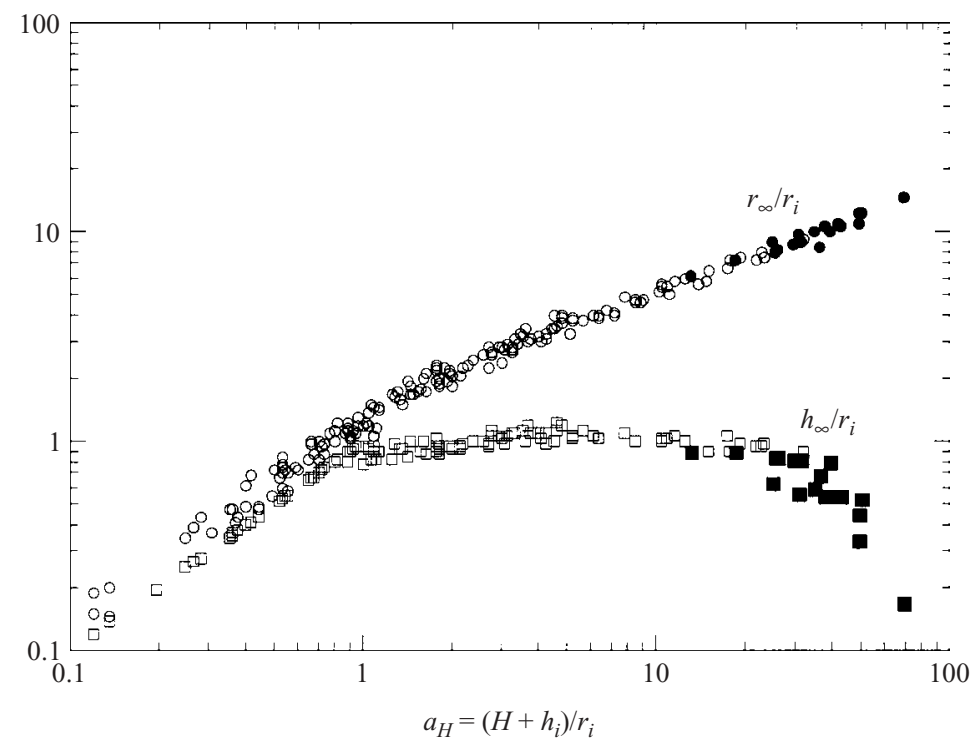

FIGURE 15. The non-dimensional final runout, $r_{\infty} / r_{i}$, and central height, $h_{\infty} / r_{i}$, as functions of the extended aspect ratio $a_{H}$ for our 'drop' experiments. The open symbols represent $H=0$ while the solid symbols represent $H \neq 0$.

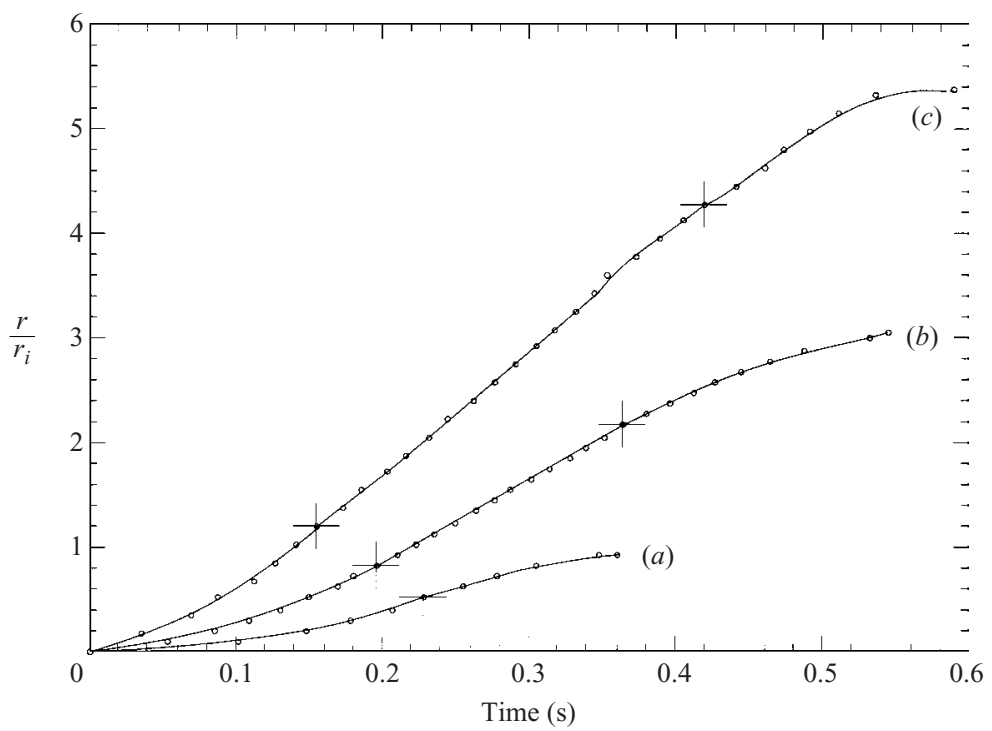

FiguRE 16. Typical radial displacements as functions of time. (a) Flows with small aspect ratio have a primary acceleration phase followed by a deceleration phase. (b) Flows with intermediate aspect ratios incorporate also a constant velocity phase. $(c)$ Flows with large aspect ratios additionally incorporate a very small deceleration phase before the constant velocity phase and a very small acceleration phase after it. The large crosses mark the ends of the acceleration phase at $\left(\tau_{f}, r_{f}\right)$ and the constant velocity phase.

followed by an intermediate phase of constant velocity and finally a deceleration phase (figure 16b). Our data suggest that the change from the acceleration phase to the phase of constant velocity coincides with the beginning of deformation of the upper surface of the column. The pattern for higher-aspect-ratio flows (figure 16c) slightly 


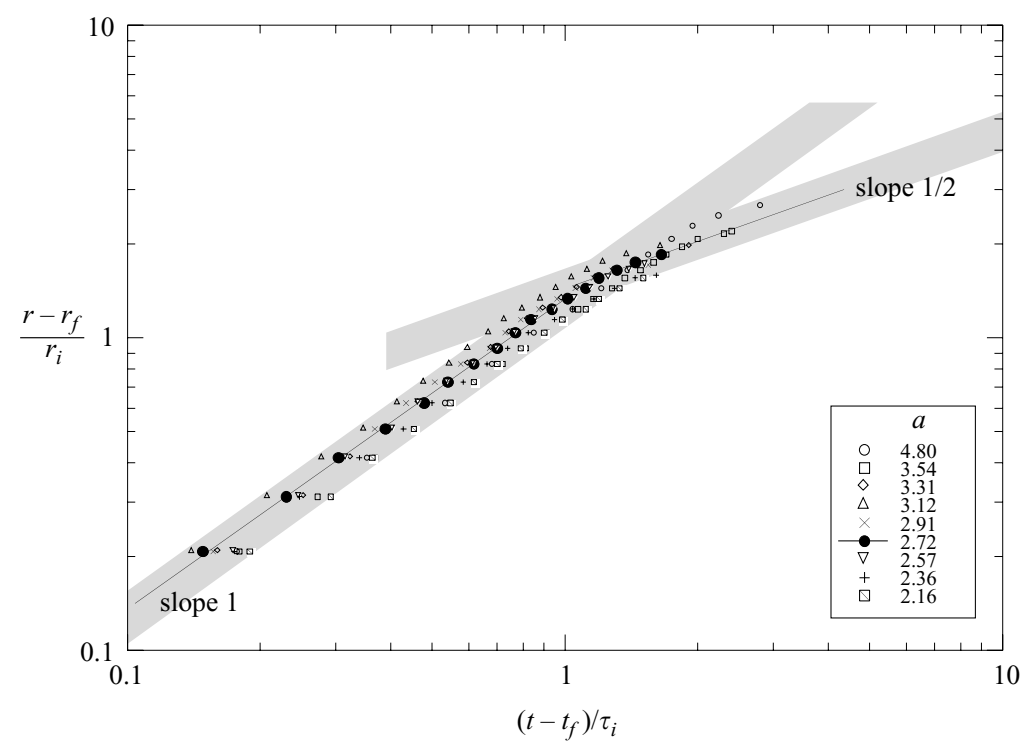

FigURE 17. The radial displacement from $r_{f}$, non-dimensionalized by $r_{i}$, as a function of the time since $\tau_{f}$, non-dimensionalized by $\tau_{i}=\pi r_{i}^{2} /\left(g V_{i}\right)^{1 / 2}$, for experiments for various $a$ with a constant-velocity phase. A clear linear phase is followed by a phase of slope $1 / 2$.

deviates from that observed for flows with intermediate aspect ratios. Before the final deceleration phase the flow front gently accelerates. Comparison of these curves with the high-speed movies suggests that the slight acceleration seems to coincide with the arrival of the wave that travels through the flow at the front.

For all flow regimes, the initial horizontal acceleration was approximately constant at between 0.25 and $0.3 \mathrm{~g}$. Further analysis is constrained due to the non-uniformities in lifting the cylinder.

It is interesting to compare the remainder of the flow to the evolution of an initially cylindrical volume of radius $r_{i}$, height $h_{i}$ (and volume $V_{i}=\pi r_{i}^{2} h_{i}$ ) of fluid of density $\rho$ propagating below a large body of fluid of small density $\rho_{1}$ (Simpson 1997; Huppert 2000). For an instantaneous release (like that considered above) there is a brief acceleration phase (which to our knowledge has not been quantitatively addressed), followed by a phase during which the front of the flow propagates at a constant velocity, while the free surface bore initiated by the removal of the confining gate propagates inward towards the centre, from where it is reflected and catches up with the propagating front (Rottman \& Simpson 1983). This constant velocity is proportional to $(g h)^{1 / 2}$, where $g$ is the acceleration due to gravity. Thereafter a similarity form of solution develops with the radial coordinate at the front $r$ proportional to $\left(g V_{i}\right)^{1 / 4} t^{1 / 2}$ (Hoult 1972). The transition between these two phases occurs on a timescale

$$
\tau_{i}=\left(g V_{i}\right)^{1 / 2} /\left(g h_{i}\right)=\pi r_{i}^{2} /\left(g V_{i}\right)^{1 / 2} .
$$

This timescale suggests analysing the motion after the acceleration phase, which is defined to end at $t_{f}, r_{f}$, by using the lengthscale $r_{i}$ and the timescale $\tau_{f}$ to graph the non-dimensional runout distance $\left(r-r_{f}\right) / r_{i}$ as a function of the non-dimensional time $\left(t-t_{f}\right) / \tau_{i}$. This is done, using logarithmic scales, in figure 17. We see that there is a clear first phase of unit slope, followed by a brief second phase of slope $1 / 2$ - before 


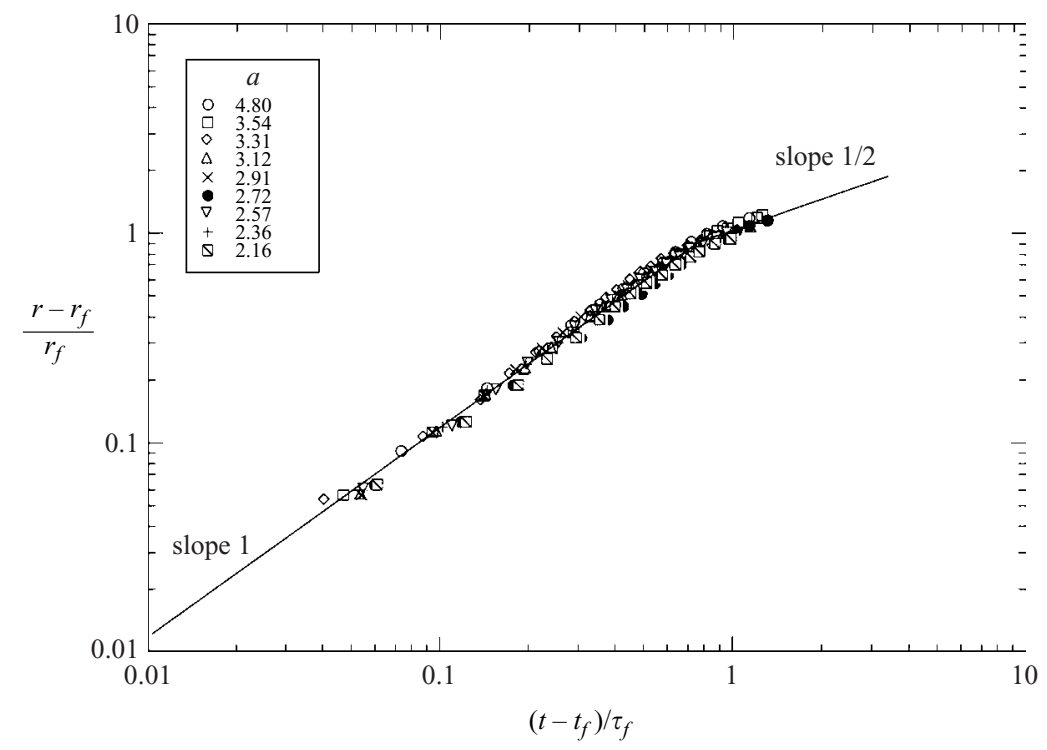

FIGURE 18. The radial displacement from $r_{f}$, non-dimensionalized by $r_{f}$, as a function of the time since $\tau_{f}$, non-dimensionalized by $\tau_{f}=\pi r_{f}^{2} /\left(g V_{i}\right)^{1 / 2}$. This non-dimensionalization collapses all the data.

the quite sudden cessation of motion. A better collapse of the data can be obtained by instead plotting $\left(r-r_{f}\right) / r_{i}$ against $\left(t-t_{f}\right) / \tau_{i}$ where $\tau_{f}=\pi r_{f}^{2} /\left(g V_{i}\right)^{1 / 2}$, as shown on figure 18. The close analogy between the forms of motion identified here and with a frictionless fluid gravity current strengthens further our argument that intergranular frictional forces play a negligible role in the spreading of granular columns (except just at the end, during the stopping phase).

From our high-speed cinematography we determined the time, $t_{\infty}$, at which the motion of the flow front ceased. By dimensional arguments $t_{\infty}$ must be of the form

$$
t_{\infty}=\left(r_{i} / g\right)^{1 / 2} G(a),
$$

for some functional form of $G(a)$. For $a<1.7$, the expression for $t_{\infty}$ must be independent of $r_{i}$ and so

$$
G(a)=K a^{1 / 2}
$$

for some constant $K$. In figure 19 we plot the data for sand, from which it is seen that there is good agreement with the functional form (15) if $K=3.0$. Somewhat surprisingly, for $a>1.7$ the data are still well represented by

$$
G(a)=3.0 a^{1 / 2}
$$

or $t_{\infty}=3.0\left(h_{i} / g\right)^{1 / 2}$. Note that the time of free-fall of a particle from a height $h_{i}$ is given by $\left(2 h_{i} / g\right)^{1 / 2} \approx 1.41\left(h_{i} / g\right)^{1 / 2}$ which indicates that the total spreading time is approximately twice the free-fall time.

We note that all the experimental flows come to an abrupt halt. Typical times between the flow front and the adjacent particles in the frontal region ceasing motion was in the order of 100 milliseconds, which represents approximately $5 \%$ of the total emplacement time. The process offreezing the flow involves rapid and sometimes 


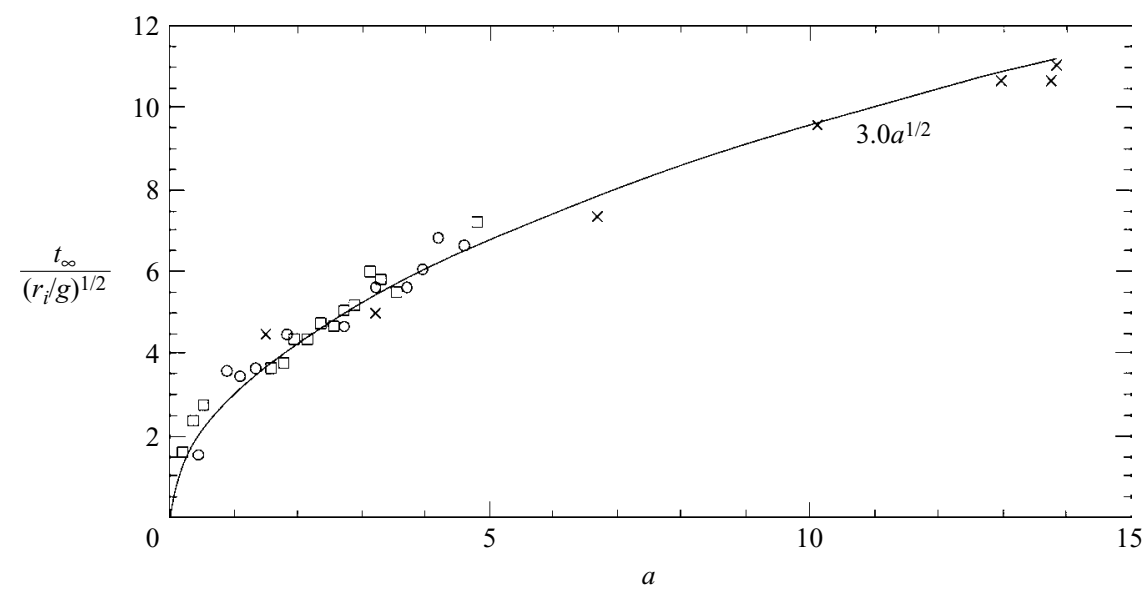

FIgURE 19. The non-dimensional time for the flow front to cease motion as a function of the aspect ratio, $a$. Initial radius $r_{i}=2.9,7.5$ and $9.7 \mathrm{~cm}$ are represented by $\times, \bigcirc$ and $\square$ respectively.

jump-like propagation of interfaces between flowing and static particles which are observed at the base and free surfaces of the flow. This behaviour contrasts with the motion of density currents of simple Newtonian fluids which flow continuously, with a transition from the inertial regime to a viscous regime of slow creeping flow (Huppert 1982). We interpret this difference as a consequence of intergranular friction becoming dominant as the flows lose energy and freely flowing granular material is converted into a static 'solid-like' granular pile.

\section{Discussion}

A common approach to understanding fluid flow phenomena is to develop fundamental theoretical analyses of the fluid forces, usually using the continuum approach. Results of model calculations using such theories can then be verified by experiments. In the case of granular materials this approach has proved difficult. The continuum approach may not be strictly valid and it has proved hard theoretically to develop models of the small-scale particle interactions and relate these to the larger-scale motion of granular materials except for extremely simplified systems. Experimental work is also complicated by the problem that steady flows of granular materials can only be established under rather special circumstances, for example down slopes where the slope is between the dynamic and static angles of friction. However, in nature and probably in most industrial situations granular flows occur in circumstances where steady flow conditions are unlikely.

These difficulties suggest that an alternative approach might help advance understanding. Here we have investigated experimentally a simple flow configuration in which a cylinder of granular material is allowed to relax to a stable configuration by slumping on a horizontal surface. Observation of the motions, flow phenomena and final configuration of the system combined with scaling arguments then provide clues to the mechanisms of granular flow. The flows can also be compared with other kinds of fluids flowing in similar configurations to give insight into the dynamics.

In the present experiments we observe that the flows are in general unsteady with three main phases being recognized: an initial acceleration; a constant-velocity phase 
(which may be absent); and a gentle deceleration followed by an abrupt halt of the flow front in typically less than $5 \%$ of the overall time for the experiment. The observation of a significant period of constant-velocity flow is particularly interesting, because this kind of behaviour is also observed for slumps of Newtonian fluids where the flow is dominated by inertia. The granular materials are effectively behaving as if they were simply dense fluids with internal friction playing no role. For a Newtonian fluid the internal friction is viscosity and for granular flows it is the frictional interactions between particles. Thus the gross behaviour of the currents in the constant-velocity phase can be described in a very similar way to a simple gravity current in the initial slumping phase. The experimental observations also indicate that during this constant-velocity stage there is very little particle deposition except for the formation of a very thin layer of particles at the base derived from the leading edge of the flow. It is clear from observations of the final position of different concentric zones in the flow that almost all of the slumped material is in motion until very close to the end.

The flows are finally emplaced in a rather short decelerating phase. The observations of the kinematics can be usefully compared to simple gravity currents of Newtonian fluids. We observe that in the decelerating phase the flows follow a power law, which is similar to that observed for inertial gravity currents of Newtonian fluids. Thus even in the third phase, shortly before they come to a halt, the flows seem to behave as inertial currents where internal friction due to particle interactions is not important. It is only at the very end that the flows depart significantly and fundamentally from behaviour consistent with a simple balance of buoyancy and inertia. In a Newtonian fluid the system would have a final transition to a viscous regime where buoyancy and viscosity are balanced. The granular flows instead come to an abrupt halt which is associated with transmission of very rapidly moving interfaces between static and moving material. These observations can be interpreted as the consequence of the flow losing energy and the rather abrupt onset of strong frictional interactions between the particles. The flow ceases motion in all places over a very short period of time $(\approx 5 \%$ of the total) and can be viewed as close to en masse freezing. A competing view of the decelerating phase is that it represents the period when frictional forces start to become important and that the similarity of the power law of distance versus time to an inertial gravity current is merely a coincidence.

The final configuration of the flow deposits is not strictly self-similar and depends on the initial geometry of the cylinder of sand. Flows with more potential energy (generally higher cylinders) can produce deposits with a conical geometry that are well below the angle of static or even dynamic friction.

These results and the ideas that derive from our observations may serve to elucidate many different features of granular flows. For example, there has been a long-running controversy about the mechanism of emplacement of pyroclastic flows. Two schools of thought have emerged as follows.

In one concept, pyroclastic flows are envisaged as dilute and turbulent particulate currents from which a deposit is formed by progressive aggradation (Branney \& Kokelaar 1992). In this model the deposit is built up layer by layer over a significant period of time, perhaps comparable to the time it takes the current to move from its source to its maximum distance or longer. In another concept, pyroclastic flows are envisaged as concentrated flows, which come to an abrupt halt by en masse freezing (Sparks 1976). The cause of freezing was originally attributed to the flow having nonNewtonian rheology, with the flow stopping abruptly when the basal shear stress falls below the yield strength. The non-Newtonian model is no longer thought to represent 
a helpful way of describing concentrated pyroclastic flow behaviour. Instead it is increasingly recognized, on the basis of observations, experiment and theory, that pyroclastic flows are better envisaged as granular flows, which may also be partially fluidized (e.g. Sparks 1976; Wilson 1980; Drake 1990; Grunewald et al. 2000; Roche et al. 2002).

Our experiments show that an alternative mechanism exists to bring granular flows to an abrupt halt. The flow stops abruptly when the particle frictional forces start to dominate. In this case it is not strictly en masse freezing as it takes a finite (but small) time for the interface between moving and deposited material to sweep through the deposit. However, this depositional time is much shorter than the emplacement time of the current and material can only move rather short lateral distances. If particles of different size and density have been sorted within the flow during transport then these arrangements might be expected to be preserved with rather limited modification within the final deposit. This is in contrast to the model of Branney \& Kokelaar (1992) where vertical variations in the final deposit reflect the fluctuations in the current properties passing a local point of deposition. This mechanism thus provides a model for pyroclastic emplacement from concentrated currents, which is rather closer to that envisaged by Sparks (1976) and Wilson (1985).

Our novel investigation immediately suggests further studies which we have already begun to consider. First, we plan to explore further the effects of raising the material to a height $H$ before it drops initially to the base, as discussed briefly in $\S 4.5$. Second, how are the various phenomena categorized and what are the quantitative results for a wide rectangular box of granular material - essentially a Cartesian, two-dimensional situation? Third, what new phenomena are introduced when the base is inclined below or above the angle of response? Fourth, how do these results differ if the spreading is into water, not air, or into a very viscous fluid, like oil? Fifth, how is the transition between a flowing granular material and a static deposit best described quantitatively? We intend to provide answers to each of these queries in subsequent publications.

We acknowledge with pleasure fruitful discussions with A. Hogg and R. Kerswell. G. L. was supported by a DAAD-scholarship and a predoctoral fellowship under the EC Volcanology Training Network (HPRN-CT-2000-00060) at Bristol. R. S. J. S. was supported by a NERC Research Professorship and a Royal Society - Wolfson merit award.

\section{REFERENCES}

ANDERson, K. G. \& JACKSON, R. 1992 A comparison of the solutions of some proposed equations of motion of granular materials for fully developed flow down inclined planes. J. Fluid Mech. 92, 145-168.

Branney, M. J. \& KokelaAR, B. P. 1992 A reappraisal of ignimbrite emplacement: changes from particulate to non-particulate during progressive aggradation of high grade ignimbrite. Bull. Volcanol. 54, 504-520.

Campbell, C. S. 1990 Rapid granular flows. Annu. Rev. Fluid Mech. 22, 57-92.

Cates, M. E., Wittmer, J. P., Bouchaud, J.-P. \& Claudin, P. 1999 Jamming and static stress transmission in granular materials. Chaos 9, 511-522.

DAde, W. B. \& Huppert, H. E. 1998 Long runout rockfalls, Geology 26, 803-806.

DAerr, A. \& Douady, S. 1999 Two types of avalanche behaviour in granular media. Nature 399, 241-243.

DraKe, T. G. 1990 Structural features in granular flows. J. Geophys. Res. 95, 8681-8696. 
DruitT, T. H. 1998 The eruption, transport and sedimentation of pyroclastic flows. In The Physics of Volcanic Eruptions (ed. J. Gilbert \& R. S. J. Sparks). Geol. Soc. London Spec. Publ. 145, $147-200$.

Freundt, A. \& Bursik, M. I. 1998 Pyroclastic flows transport mechanisms. In From Magma to Tephra: Modelling Physical Processes of Explosive Volcanic Eruptions (ed. A. Freundt \& M. Rosi). Developments in Volcanology, vol. 4, pp. 173-245. Elsevier.

DE Gennes, P. G. 1999 Granular matter: a tentative view. Rev. Mod. Phys. 71, 5374-5382.

Grunewald, U., Sparks, R. S. J., Kearns, S. \& Komorowski, J. C. 2000 Friction marks on blocks from pyroclastic flows at the Soufriere Hills volcano, Montserrat: Implications for flow mechanisms. Geology 28, 827-830.

Hoult, D. P. 1972 Oil spreading on the sea. Annu. Rev. Fluid Mech. 4, 341-368.

HuPPERT, H. E. 1982 The propagation of two-dimensional and axisymmetric viscous gravity currents over a rigid horizontal surface. J. Fluid Mech. 121, 43-58.

Huppert, H. E. 1998 Quantitative modelling of granular suspension flows. Phil. Trans. R. Soc. Lond. 356, 2471-2496.

Huppert, H. E. 2000 Geological Fluid Mechanics. In Perspectives in Fluid Dynamics: A Collective Introduction to Current Research (ed. G. K. Batchelor, H. K. Moffatt \& M. G. Worster), pp. 447-506. Cambridge University Press.

Iverson, R. M. \& Denlinger, R. P. 2001 Flow of variably fluidized granular masses across three-dimensional terrain. Part I: Coulomb mixture theory. J. Geophys. Res. 106, 537-552.

Jaeger, H. M., Nagel, S. R. \& Behringer, R. P. 1996 Granular solids, liquids, and gases. Rev. Mod. Phys. 68, 1259-1273.

Kadanoff, L. P. 1999 Built on sand: Theoretical ideas inspired by granular flows. Rev. Mod. Phys. 71, 435-444.

Pouliquen, O. 1999 Scaling laws in granular flows down rough inclined planes. Phys. Fluids 11, $542-548$.

Pouliquen, O. \& Forterre, Y. O. 2002 Friction law for dense granular flows: application to the motion of a mass down a rough inclined plane. J. Fluid Mech. 453, 133-151.

Roche, O., Gilbertson, M., Phillips, J. \& Sparks, R. S. J. 2002 Experiments on gas-fluidized granular flows and implications for pyroclastic flow mobility. Geophys. Res. Lett. 29, 40-44.

RotTman, J. W. \& Simpson, J. E. 1983 Gravity currents produced by instantaneous releases of a heavy fluid in a rectangular channel. J. Fluid Mech. 135, 95-110.

Savage, S. B. 1995 Flows of granular materials. Proc. CANCAM '95 (ed. B. Tabarrok \& S. Dost), pp. 62-73. University of Victoria, BC, Fleming Express Press Ltd, Victoria, BC.

SAvage, S. B. 1998 Analyses for slow quasi-static, high concentration flows of granular materials. J. Fluid Mech. 377, 1-26.

Savage, S. B. \& Hutter, K. 1989 The motion of a finite mass of granular material down a rough incline. J. Fluid Mech. 199, 177-215.

Simpson, J. E. 1997 Gravity Currents in the Environment and the Laboratory, 2nd Edn. Cambridge University Press.

Sparks, R. S. J. 1976 Grain size variations in ignimbrites and implications for the transport of pyroclastic flows. Sedimentology 23, 147-188.

Wilson, C. J. N. 1980 The role of fluidisation in the emplacement of pyroclastic flows: an experimental approach. J. Volcanol. Geoth. Res. 8, 231-249.

Wilson, C. J. N. 1985 The Taupo eruption, New Zealand II. The Taupo ignimbrite. Phil. Trans. R. Soc. Lond. A 314, 229-310. 